\title{
CIC missense variants contribute to susceptibility for spina bifida
}

\author{
Xiao $\mathrm{Han}^{1}$, Xuanye $\mathrm{Cao}^{2}$, Vanessa Aguiar-Pulido ${ }^{3}$, Wei Yang ${ }^{4}$, Menuka Karki², Paula \\ Andrea Pimienta Ramirez ${ }^{2}$, Robert Cabrera ${ }^{2}$, Ying Lin², Bogdan Wlodarczyk ${ }^{2}$, Gary \\ Shaw $^{4}$, Margaret Ross ${ }^{5}$, Cuilian Zhang ${ }^{1}$, Richard Finnell ${ }^{2}$, and Yunping Lei ${ }^{2}$ \\ ${ }^{1}$ Henan Provincial People's Hospital \\ ${ }^{2}$ Baylor College of Medicine \\ ${ }^{3}$ Weill Cornell Medical College \\ ${ }^{4}$ Stanford University School of Medicine \\ ${ }^{5}$ Weill Cornell Medicine
}

November 30, 2021

\begin{abstract}
Neural Tube Defects (NTDs) are congenital malformations resulting from abnormal embryonic development of the brain, spine, or spinal column. The genetic etiology of human NTDs remains poorly understood despite intensive investigation. CIC, homolog of the Capicua transcription repressor, has been reported to interact with ataxin-1 (ATXN1) and participate in the pathogenesis of spinocerebellar ataxia type 1. Our previous study demonstrated that CIC loss of function (LoF) variants contributed to cerebral folate deficiency by downregulating folate receptor 1 (FOLR1) expression. Given the importance of folate transport in neural tube formation, we hypothesized that CIC variants could contribute to increased risk for NTDs by depressing embryonic folate concentrations. In this study, we examined CIC variants from whole genome sequencing (WGS) data of 140 isolated spina bifida cases and identified 8 missense variants of CIC gene. We tested the pathogenicity of the observed variants through multiple in vitro experiments. We determined that CIC variants decreased FOLR1 protein level and planar cell polarity (PCP) pathway signaling in a human cell line (HeLa). In a murine cell line (NIH3T3), CIC loss of function variants down regulated PCP signaling. Taken together, this study provides evidence supporting CIC as a risk gene for human NTD.
\end{abstract}

\section{INTRODUCTION}

Neural Tube Defects (NTDs) are severe congenital malformations in which the neural folds that ultimately give rise to the spine and brain failed to properly close during embryonic development. Spina bifida and anencephaly are the two most common subtypes of NTDs that affect the spinal cord and brain, respectively (Greene \& Copp, 2014). Given their severity, NTDs are the leading cause of death in the first year of life. Standard of care for NTD varies greatly across nations. Including anencephaly, which is uniformly fatal, over $75 \%$ of NTD-affected births resulted in death before 5 years of age (Blencowe, Kancherla, Moorthie, Darlison \& Modell, 2018). In the industrialized nations, surgical and medical management has improved to the point that the majority of spina bifida affected infants will survive into adulthood (Oakeshott, Hunt, Poulton \& Reid, 2010), but will suffer long-term associated disabilities and treatment costs remain major obstacles (Flores, Vellozzi, Valencia \& Sniezek, 2014). The worldwide prevalence of NTDs ranges from 1 to 10 per 1000 births, varying widely among different countries and regions. The annual worldwide prevalence of NTD-affected birth was estimated to exceed 260,000 births (Blencowe et al. 2018; Au et al. 2010).

NTDs are complex malformations which have multifactorial etiologies, including genetic, environmental and dietary factors (CoppJ \& Greene, 2013). There is both clinical and experimental evidence that maternal folate status is a nutritional modifier of NTD risks (Caffrey, McNulty, Irwin, Walsh \& Pentieva, 2019). Folate molecules participate in one carbon metabolism to provide thymidine and purines for DNA synthesis and 
s-adenosyl methionine (SAM), which is the universal methyl group donor required for multiple methylation reactions (Froese Fowler \& Baumgartner, 2019). Up to $70 \%$ of NTDs may be folate-sensitive and can be prevented by folic acid supplementation prior to conception and continued in early pregnancy (Shlobin, LoPresti, Du \& Lam, 2020). Based on this, the United States Food and Drug Administration (FDA) has required mandatory folic acid fortification of grain products including flour, bread, pasta, rice, and cereal in 1998. The United States Centers for Disease Control and Prevention (CDC) has also urged every woman who may become pregnant to obtain at least 400ug of folate every day to prevent NTDs occurrence.

Genetic factors are also considered to be significant contributors to the occurrence of NTDs in both human and mice (Copp \& Greene, 2010; Wallingford, Niswander, Shaw \& Finnell, 2013; Wilde, Petersen \& Niswander, 2014; Greene, Stanier \& Copp, 2009). Several studies using Splotch mouse mutant have shown that NTDs can be related back to their compromised ability to maintain cellular proliferation and an undifferentiation state of their neuroepithelium which hampers normal neurulation and leads to the failure of neural tube closure. Folic acid supplementation restores proper proliferation in the cranial neuroepithelium and compensated for the loss of Pax3, which prevents cranial NTDs and thereby rescues the normal phenotype (Sudiwala et al. 2019). Mutations in the Vangl2 gene cause craniorachischisis through impaired Vangl2 interaction with Dvl1, Dvl2, and Dvl3 (Torban, Wang, Groulx \& Gros, 2004). Furthermore, NTD caused by point mutation in the $\mathrm{Lrp} 6^{\mathrm{Cd} / \mathrm{Cd}}$ canonical WNT coreceptor are rescued both by folic acid and by inhibition of the WNT non-canonical pathway by RhoA inhibitors (Gray et al., 2013; Carter, Ulrich, Oofuji, Williams \& Elizabeth Ross, 1999). To date, mutations in more than 300 genes have been reported to cause NTDs in mice (Wilde, Petersen \& Niswander, 2014). Multiple signaling pathways, including the Wnt/planar cell polarity (PCP) pathway (Chen et al., 2018), sonic hedgehog (SHH) pathway (Murdoch \& Copp, 2010) and mitochondrial folate metabolic pathway (Kim et al., 2018), have been reported to be involved in neural tube closure. However, only a few of these reported murine NTD genes have been positively associated with human NTDs, as the pattern of variants reported in human NTD patients supports a polygenic or oligogenic etiology (Copp \& Greene, 2010).

CIC, homolog of the Drosophila capicua gene, was discovered in a screen for mutations affecting the anteroposterior pattern of Drosophila embryos (Bettegowda et al., 2011). It was found to function downstream of the receptor tyrosine kinase (RTK) pathways that includes the epidermal growth factor receptor, Torso, Ras, Raf, and mitogen-associated protein kinases (MAPKs), which are all related to embryonic pattern formation (Roch, Jiménez \& Casanova, 2002). CIC can be transported into the nucleus through interactions with Karyopherin Subunit Alpha 3 (KPNA3) and serves as a transcription repressor. In the absence of EGFR-ERK signaling, CIC binds to target promoters and/or enhancers and represses downstream genes, while activation of the pathway leads to CIC degradation and activation of genes normally repressed by CIC (Jiménez, Shvartsman \& Paroush, 2012; Astigarraga et al., 2007).

Recurrent mutations in CIC were initially identified in oligodendroglioma; subsequently, several CIC aberrations were found in multiple types of cancers including medulloblastoma, breast cancer, and small blue round cell tumors (Wong \& Yip, 2020; Huang et al., 2016). In mammals, CIC forms a transcriptional repressor complex with ATXN1. Gain of function of the complex underlies the pathogenesis of spinocerebellar ataxia type 1 (Lam et al., 2006), and a spectrum of neurobehavior phenotypes which include hyperactivity, impaired learning and memory, and abnormal maturation and maintenance of upper-layer cortical neurons (Lu et al., 2017). Our previous study demonstrated that CIC acts as a transcription activator of FOLR1 and CIC loss of function contributes to the occurrence of cerebral folate deficiency through diminished FOLR1 expression (Cao et al., 2021). Although previous studies have identified mutations in the human CIC gene are associated with neurobehavioral phenotypes, there are no reports of CIC mutations in NTD patients.

To better understand the genetic etiology of human NTDs, we analyzed whole genome sequencing (WGS) data on 140 cases with isolated spina bifida from the U.S. and detected eight rare missense CIC variants. Functional analysis indicated that CIC missense variants identified in NTD cases downregulated the FOLR1 protein level and PCP signaling in Hela and NIH3T3 cell lines. Overall, our results support for the first time that CIC variants potentially contribute to the etiology of human NTDs. 


\section{MATERIALS AND METHODS}

\section{Human subject}

A total of 140 case samples with isolated spina bifida (myelomeningocele) which recently reported in our paper (Wolujewicz et al., 2021) were collected from California, USA and Doha, Qatar. Cases with no other anomalies, only minor anomalies or related anomalies were considered isolated.

\section{Editorial Policies and Ethical Considerations}

The study was approved by institutional review board of Baylor College of Medicine, Stanford University, Weill Medical College of Cornell University and Hamad Medical Corporation. All samples were collected with written informed parental consent. CIC variants from gnomAD database were used as controls.

\section{Sequencing analysis of NTD samples}

The WGS data were processed using standard pipelines, as described in the Broad Institute's GATK Best Practices (Van der Auwera et al., 2013). Reads were aligned to the hg38 reference provided as part of the GATK Bundle using BWA (Li \& Durbin, 2009). Variant calling was performed with GATK4 (Poplin et al., 2018) and joint genotyping was carried out on the whole cohort, followed by Variant Quality Score Recalibration (VQSR). Quality control (following standard practices such as obtaining sequencing metrics, per sample missing rate and level of heterozygosity), was done to check for DNA contamination and identify outliers, removing those samples with poor quality. Per-variant quality was also assessed and only variants with a "PASS" in the filter column were retained and annotated utilizing Ensembl Variant Effect Predictor (VEP) v.95 (McLaren et al., 2016). GnomAD (https://gnomad.broadinstitute.org/ ) database v2.1.1 was used as a reference to determine whether the variant is novel (allele frequency $(\mathrm{AF})=0$ ) or is rare (AF $<0.001)$. Pathogenic effect prediction of all missense variants were performed using the online program SIFT (Sorting Intolerant From Tolerant;https://sift.bii.a-star.edu.sg). All parameters were set as per the software's default settings. The localization of the variants in their protein domains was assessed by Uniprot (http://www.uniprot.org/ ). Gene lollipop structure was plotted using Lollipops program (Jay \& Brouwer, 2016).

All eight CIC variants passed Bamfile checking (IGV) and were then validated by Sanger Sequencing. Variants lollipop plot was generated using the Lollipops software (Rothenberg et al., 2004).

\section{Plasmids}

Wildtype EGFP-CIC plasmid and the R353X EGFP-CIC mutant plasmid were reported in our previous paper (Cao et al., 2021). Variants identified in NTDs were introduced into wildtype EGFP-CIC plasmid using site direct mutagenesis method by Genescript company.

Cell culture and transfection Hela cells and NIH3T3 cells were grown in Dulbeco's Modified Eagles's Medium (DMEM, Sigma, D6429), supplemented with 10\% heat-inactivated fetal bovine serum (FBS, Gibco) and 1X Antibiotic-Antimycotic (Gibco). NIH3T3 cells were cultured on $0.1 \%$ Gelatin (Gibco)-coated plate. Cell cultures were maintained at 37 in a humidified atmosphere containing $5 \% \mathrm{CO}_{2}$. Transfection was performed at 50-70\% confluency using Lipofectamine 2000 (Invitrogen) according to the manufacturer's protocol. Medium was replaced with the culture medium without antibiotics prior to transfection. Further experiments were performed 24-48 hours after transfection.

\section{Immunofluorescence staining}

Hela cells for immunofluorescence staining were plated on glass bottom dishes. 24-48 hours after transfection, cells were fixed with pre-warmed $4 \%$ paraformaldehyde for 30 minutes at room temperature. Cells were permeabilized with $0.3 \%$ Triton X-100 in TBST for 20 minutes at room temperature, and then were blocked with 10\% NGS in TBST for 1 hour at room temperature. FOLR1 antibody (1:100, Proteintech, 23355-1-AP) was diluted in blocking buffer and incubate overnight at 4. 14-16 hours later, cells were rinsed with PBS and incubated with secondary antibody (1:1000, Cell Signaling Technology, 8889S) diluted in blocking buffer for 
1 hour in the dark. Cells were rinsed with PBS and allowed to air dry. A drop of antifade mounting medium with DAPI (Invitrogen, P36931) was added to the slides which were covered with a coverslip. Images were taken using a deconvolution microscope (Nikon T2).

\section{Western Blotting}

Cells for western blotting were plated on 6 -well plates (Corning). 48 hours post-transfection, Hela cells and NIH3T3 cells were rinsed with ice-cold PBS, collected with cell scrapers and lysed with RIPA buffer (Thermo Scientific, 89900) with protease inhibitor (Sigma, 11836170001) for 30 minutes on ice, and were then centrifuged at 14000rpm for 20 minutes. The supernatant was transferred to a new tube to which SDS loading buffer was added and mixed before being boiled for 10 minutes at 95 . Western Blotting was then performed as previously described. The proteins were immunoblotted with GFP antibody (1:1000, Santa Cruz Biotechnology, sc-9996), FOLR1 antibody (1:1000, Invitrogen, PA5-86666), GAPDH antibody (Cell Signaling Technology, 5174S), Vangl2 antibody (1:1000, Proteintech, 21492-1-AP), RhoA antibody (1:500, Santa Cruz Biotechnology, sc-418) and CIC antibody (Cat\#: A301-204A, Bethyl, TX) overnight at 4. On the second day, the membrane was incubated with HRP-conjugated anti-rabbit antibody or anti-mouse antibody (1:5000, Cell Signaling Technology, 7074S and 7076S) for 1 hour at room temperature. Membranes were rinsed with ECL substrates (Thermo Scientific, 34580) and protein bands were detected using a BioRad Gel Doc XR+ Imaging System.

\section{Folate binding assay and folate quantification}

Hela cells were passaged and cultured in RPMI 1640 folate-free media (Sigma, R1145), supplemented with 10\% FBS, 3.7g/L sodium bicarbonate, 1X glutamax (Gibco, 35050-061) and 1X Antibiotic-Antimycotic. Cells were transfected with wildtype and variant GFP-CIC plasmids when reaching $50 \%$ confluency. 36-48 hours later, culture medium was replaced with PBS supplemented with $2 \%$ FBS and 50nM folic acid (Sigma, F8758). Cells were cultured for 1 hour in the presence of FA, after which they were dissociated with $0.25 \%$ trypsin. Dissociated cells were resuspended in TBST with $1 \%$ ascorbic acid $(\mathrm{pH} \mathrm{3.0-3.5)}$ and incubated at 95 for 10 minutes. Supernatant was collected after being centrifuged for 10 minutes at $14000 \mathrm{rpm}$. NaOH $(1 \mathrm{M})$ was added to collected supernatant at a ratio of 1:15 to reach a neutral $\mathrm{pH}$ value.

Bovine folate binding protein (50ng/ul, Sigma, F0504-1MG) was printed on a 96 well plate with $1 \mathrm{ul}$ in each well, and the plate was then sealed and incubated overnight at 4. On the second day, HRP-FA was 2-fold serially diluted to create a standard curve. Samples and HRP-FA were mixed at a ratio of 4:1. The printed 96 well plate was washed with TBST, and the mixed samples and standards were added into the wells and incubated for 1 hour prevented from light. The plate was washed with TBST, and 50ul of ELISA substrate were added to each well. Signal intensities were imaged using Q-view equipment and quantified using the Q-view software.

\section{Statistical analysis}

All data were analyzed with a Student's t-test, and P-values $<0.05$ were considered statistically significant. Data are presented as mean +- SE of the mean. All data were analyzed using two-tailed t-test.

\section{RESULTS}

\section{Rare missense variants in CIC are associated with human NTD risks}

WGS was performed on DNA samples from 140 spina bifida cases. Eight rare missense variants (allele frequency $<0.001$ in gnomAD) in CIC gene were identified in 140 individuals (Table 1). Seven of the eight missense variants were predicted to be damaging using Polyphen or SIFT software, indicating they are likely pathogenic mutations. Lollipop plots showed that none of the eight missense mutations locates in the HMG box region (Fig. 1A). All eight variants were evolutionarily conserved across multiple mammalian species (Fig. 1B). Among these missense variants, p. E1452D and p. M671V were absent in the gnomAD database, indicating that they are novel variants. Notably p. M671V is also predicted-to-be-damaging by both prediction software and locates in a highly conserved region. All eight variants were heterozygous in 
the seven different infants with isolated spina bifida (myelomeningocele). Three CIC variants carriers were collected from California before folic acid fortification. One case carried biallelic CIC variants (p.R666H and p.R1205Q).

\section{CIC missense variants affected protein expression levels but not localization}

To determine whether the variants affect CIC protein subcellular localization or their level of expression, functional analyses were performed on the CIC variants. As shown in Fig. 2A, wildtype GFP-CIC was primarily located in the nucleus as expected. All eight variants were also located in the nucleus, indicating that none of these variants affected the cellular localization of CIC protein. Interestingly, we noticed that some of the mutants showed a weaker nuclear staining under the same exposure conditions. We speculated that the expression level of CIC may be affected by the missense mutations. We subsequently performed western blotting assays to investigate the effect of these variants on the CIC protein levels. As shown in Figure 2B, GFP-CIC protein of all eight variants were less abundant compared to the wildtype (pi0.05), which demonstrated that the missense variants may decrease CIC protein expression or potentially affect protein stability.

\section{CIC missense variants affected FOLR1 protein level and cellular folate concentration}

We have previously found that CIC is capable of binding to FOLR1 promoter region, thereby, regulating its transcription (Cao et al., 2021). FOLR1 is a critically important transport molecule involved in the uptake of folates into the cells and plays a crucial role in neural tube closure. In mice, Folr1 knock-out lead to 100\% penetrant spina bifida. In humans, functional disruption of FOLR1 causes cerebral folate deficiency syndrome (CFD), while increased titers of FOLR1 autoantibody in maternal serum was reported to be associated with both NTDs (Rothenberg et al., 2004; Cabrera, et al., 2008) and CFD (Ramaekers et al., 2005). We examined the effect of variant containing CIC on FOLR1 protein levels in Hela cells. As presented in Figure 3A, wildtype GFP-CIC increased FOLR1 expression compared to the control pEGFP plasmid (pi0.001), while overexpression of all GFP-CIC mutants profoundly downregulated FOLR1 expression compared to wildtype $(\mathrm{p} ; 0.01)$.

We further investigated the effect of the CIC variants on folate absorption ability in HeLa cells. As expected, wildtype CIC overexpression increased intracellular folate concentrations compared to the pEGFP control, while overexpression of CIC mutants reduced cellular folate content compared to wildtype $\left(\mathrm{p}_{i} 0.05\right)$ (Fig. 3C), indicating that CIC mutants can reduce folate binding and/or absorption ability by downregulating FOLR1.

\section{CIC missense variants downregulated PCP pathway protein Vangl2 and RhoA}

The PCP signaling pathway has been shown to play an important role in neural tube closure in multiple previous studies (Copp, Greene \& Murdoch, 2003; Wallingford, 2006; Wallingford, 2012; Murdoch et al., 2014).Genetic defects in the Vangl2 gene have been strongly associated with NTDs in mouse models, and there is growing evidence implicating Vangl genes (Vangl1 and Vangl2) in the genetic etiology of human NTDs (Kibar et al., 2007; Lei et al., 2010; Kibar et al., 2011; Tian et al., 2020). To investigate whether dysregulation of the PCP pathway also occurs in CIC variants associated spina bifida, we performed western blotting to examine the level of the PCP signaling pathway core protein Vangl2 and PCP downstream protein RhoA using overexpression wildtype CIC and CIC mutants in HeLa cells. As shown in Fig. 4, VANGL2 and RhoA protein levels in the wildtype CIC overexpression group were significantly increased compared to the pEGFP control (p;0.01), while VANGL2 and RhoA protein levels in those groups with overexpressed CIC mutants were less abundant compared to wildtype ( $\mathrm{p}_{\mathrm{i}} 0.05$ and $\mathrm{p} ; 0.01$, respectively). Protein levels of RhoA in each sample were consistent with those of Vangl2. We further investigated the effects of CIC LoF mutation on PCP pathway proteins Vangl2 and RhoA in NIH3H3 cells that were overexpressed with wildtype CIC, CICR353X and pEGFP control. As depicted in Fig. 5, both Vangl2 and RhoA showed increased protein levels in GFP-wildtype CIC transfection compared to EGCPC1 basic control vector transfection, and decreased protein level in CIC-R353X group compared to $\mathrm{WT}\left(\mathrm{p}_{\mathrm{i}} 0.01\right)$. These results support that CIC variants may also modify NTD risk through PCP signaling pathway. 


\section{DISCUSSION}

Previous studies have indicated that CIC interaction with ATXN1 contributes to the pathogenesis of spinocerebellar ataxia type 1 (Rousseaux et al., 2018). Disruption of CIC-ATXN1 complex results in hyperactivity, impaired learning and memory, and abnormal maturation and maintenance of upper-layer cortical neurons in Emx1-cre mice (Cao et al., 2021). Forebrain-specific deletion of CIC in Foxg1-cre mice caused abnormal increases in oligodendrocyte progenitor and immature oligodendrocytes populations (Yang et al., 2017). Brain-specific deletion of CIC compromised neuroblasts transition to immature neurons in mouse hippocampus and compromises normal neuronal differentiation (Hwang et al., 2020). While CIC deletion clearly impacts numerous aspects of neurodevelopment, ours is the first evidence indicating a potential relationship between CIC and neural tube development.

In the present study, we performed and support the first genetic association analysis between CIC variants and an increased risk for NTDs. We also explored the possible underlying mechanisms by which CIC could contribute to NTDs in humans. We initially focused on the folate receptors, as they are membrane proteins that mediate cellular uptake of folates. FOLR1 is important for neural tube closure during early embryogenesis, as inactivation of this gene leads to embryoic death and NTDs in mice which can be rescued by folate supplementation (Piedrahita et al., 1999). In humans, homozygous biallelic LoF FOLR1 mutations lead to extremely low 5-methyltetrohydrofolate level in the cerebrospinal fluid (CFD) but have not been observed to result in NTDs (Steinfeld et al., 2009). However, it has been reported that increased level of maternal serum FOLR1 autoantibody during pregnancy is associated with the occurrence of fetal NTDs (Rothenberg et al., 2004). Although evidence is lacking to show that isolated FOLR1 gene variants are associated with human NTDs, Saitsu (2017) found that altered spatial and temporal Folr1 expression patterns in mice are associated with anterior neural tube closure, and this expression pattern is conserved between human and mice engineered to express a lacZ reporter transgene. Additionally, there have been studies of human folate transport genes that described 12 novel variants in FOLR1, FOLR2, and FOLR3 (Findley et al., 2017) found in NTD cases. This included four large insertion deletion variants in FOLR3 as well as a single stop gain variant. While far from being conclusive evidence, it is suggestive that FOLR1 abnormalities might be involved in a subset of human NTDs. According to our previous study of CIC and CFD, CIC could regulate FOLR1 expression through binding to its promoter region. In this study, we confirmed that CIC variants can decrease FOLR1 expression levels in human cell lines. While we previously found that CIC binding motifs lie within the human FOLR1 promoter region, they do not exist in the mouse Folr1 promoter region.

Convergent extension $(\mathrm{CE})$ is a crucial process during neural tube closure by which the neural plate undergoes narrowing along its mediolateral axis and extends along anteroposterior axis (Tada \& Heisenberg, 2012). The progression of convergent extension is driven by planar polarized cell intercalation, which in turn is reported to be driven by subcellular processes including extension of mediolaterally directed cellular protrusions and shrinkage of mediolaterally oriented cell-cell junctions (Butler \& Wallingford, 2018; Blankenship, Backovic, Sanny, Weitz \& Zallen, 2006). The PCP signaling pathway is the most well-characterized regulator of cell intercalation (Butler \& Wallingford, 2017). Several genes in the PCP pathway have been associated with NTDs (Humphries, Narang \& Mlodzik, 2020). Over 300 genes are known to be causative of NTDs in mice, and many of them participate in the PCP pathway (Wang etal., 2018). Core PCP genes are highly conserved from invertebrates to mammals, among which Van Gogh (Vang), Vangl1/2 in mammals, is the critical regulator for normal extension conversion. Other PCP genes include FZD3/FZD6, CELSR1/CELSR2/CELSR3, DVL1/DVL2/DVL3, PRICKLE1/PRICKLE2/PRICKLE3/PRICKLE4 and ANKRD6. Vangl2 was the first genetically mapped PCP gene through studying Loop-tail mutant mice. Homozygous Vangl2 mutants cause craniorachischisis, the most severe type of NTD (Kibar et al., 2001), while Vangl1 and Vangl2 compound heterozygous mice also exhibit a craniorachischisis phenotype (Torban et al., 2008). Besides Vangl2, inactivation of Dvl1 and Dvl2, Celsr1, and Fzd3 or both Fzd3 and Fzd6 also lead to severe NTD phenotypes, primarily craniorachischisis and exencephaly in mice (Kibar et al., 2001; Wang et al., 2006; Ybot-Gonzalez et al., 2007; Curtin et al., 2003). In addition to these core PCP genes, some noncore PCP genes also exhibit severe NTD in mice when gene targeted, such as protein tyrosine kinase 7 (PTK7), scribbled PCP protein, the gene responsible for the circle tail mouse phenotype, Scrib, and dishevelled binding antagonist of beta- 
catenin 1 (Dact-1) (Mohd-Zin, Marwan, Abou Chaar, Ahmad-Annuar \& Abdul-Aziz, 2017). In humans, PCP genes have been examined in case-control association studies or directly sequenced in mutation screens (Juriloff \& Harris, 2012). Vangl2 variants in human NTD samples were reported by Lei et al.(2010) and Kibar et al.(2011). In the present study, we found that all eight CIC variants lead to decreased Vangl2 protein levels in human cell lines, and the CIC LoF variant also limited Vangl2 protein levels in a murine cell line. We suspect this contributes to the observed NTD phenotype.

Acting downstream of Vangl2, RhoA plays a crucial role in regulating cell shape and movement (Oishi, Makita, Sato \& Iiri, 2012). In this study, protein levels of Vangl2 and RhoA showed similar trends for different CIC variants among groups in both human and mouse cell lines. Our in vitro assays showed that CIC variants diminished both FOLR1 expression level and PCP signals, both of which could increase the likelihood of human NTDs. However, the relationship between inhibiting Folr1 and associated PCP pathway gene expression and the underlying mechanisms by which CIC affects Vangl2, remain unclear. Balashova, Visina and Borodinsky (2017) found that FOLR1 is enriched in the apical surface of the neural plate in Xenopus laevis, colocalized with C-cadherin and $\beta$-catenin, and that FOLR1 is necessary for neural plate cell apical constriction during Xenopus neural tube formation. The seemingly contradictory evidence emphasizes the importance of continued experimentation on this system.

In this study, we identified a novel gene contributing to human NTDs. We demonstrated the possible underlying mechanisms. Future studies are needed to further elucidate these cellular and molecular mechanisms, which will help us to better understand the genetic etiology of NTDs and develop effective strategies to prevent these severe birth defects.

\section{ACKNOWLEDGMENTS}

We thank the Qatar National Spina Bifida Clinic, Hamad Medical Corporation and WCM-Q, for invaluable efforts toward patient enrollment. We thank the California Department of Public Health Maternal Child and Adolescent Health Division for providing data. Findings and conclusions herein are those of the authors and do not necessarily represent the official position of the California Department of Public Health. The authors acknowledge the Texas Advanced Computing Center at The University of Texas at Austin for providing high-performance computing resources that have contributed to the research results reported within this paper. URL: http://www.tacc.utexas.edu .

\section{COMPETING INTERESTS}

R.H.F., B.W.J. and R.M.C. formerly held leadership positions with the now dissolved TeratOmic Consulting LLC. R.H.F. also receives travel funds to attend editorial board meetings of the Journal of Reproductive and Developmental Medicine published out of the Red Hospital of Fudan University. The other authors declare no competing interests.

\section{DATA AVAILABILITY}

Data pertaining to specific variants generated during the downstream analyses, which support the findings of this study, are available upon request to the corresponding author (R.H.F). De-identified data will be made available upon request.

\section{AUTHOR CONTRIBUTIONS}

X.H., X.C., Y.L., B.J.W and R.H.F. conceived and designed the experiments. Y.L., X.C. and V.A.P. generated and analyzed sequencing data. X.H., X.C., R.M.C., P.A.P.R and M.K performed cell-based functional analysis. W.Y., G.M.S., L.L.W., A.R., M.E.R., and R.H.F. identified NTD cases and acquired clinical data. X.H., X.C., Y.L., and R.H.F. drafted the manuscript, and all other authors assisted in editing.

\section{References}

1. Greene, N. D., \& Copp, A. J. (2014). Neural tube defects. Annual review of neuroscience, 37, 221-242. 
2. Blencowe, H., Kancherla, V., Moorthie, S., Darlison, M. W., \& Modell, B. (2018). Estimates of global and regional prevalence of neural tube defects for 2015: a systematic analysis. Annals of the new York Academy of Sciences, 1414(1), 31-46.

3. Oakeshott, P., Hunt, G. M., Poulton, A., \& Reid, F. (2010). Expectation of life and unexpected death in open spina bifida: a 40-year complete, non-selective, longitudinal cohort study. Developmental Medicine \& Child Neurology, 52(8), 749-753.

4. Flores, A. L., Vellozzi, C., Valencia, D., \& Sniezek, J. (2014). Global burden of neural tube defects, risk factors, and prevention. Indian journal of community health, 26(Suppl 1), 3.

5. Au, K. S., Ashley-Koch, A., \& Northrup, H. (2010). Epidemiologic and genetic aspects of spina bifida and other neural tube defects. Developmental disabilities research reviews, 16(1), 6-15.

6. Copp, A. J., \& Greene, N. D. (2013). Neural tube defects - disorders of neurulation and related embryonic processes. Wiley Interdisciplinary Reviews: Developmental Biology, 2(2), 213-227.

7. Caffrey, A., McNulty, H., Irwin, R. E., Walsh, C. P., \& Pentieva, K. (2019). Maternal folate nutrition and offspring health: evidence and current controversies. Proceedings of the Nutrition Society, 78(2), 208-220.

8. Froese, D. S., Fowler, B., \& Baumgartner, M. R. (2019). Vitamin B12, folate, and the methionine remethylation cycle-biochemistry, pathways, and regulation. Journal of inherited metabolic disease, 42(4), 673-685.

9. Shlobin, N. A., LoPresti, M. A., Du, R. Y., \& Lam, S. (2020). Folate fortification and supplementation in prevention of folate-sensitive neural tube defects: A systematic review of policy. Journal of Neurosurgery: Pediatrics, 27(3), 294-310.

10. Copp, A. J., \& Greene, N. D. (2010). Genetics and development of neural tube defects. The Journal of Pathology: A Journal of the Pathological Society of Great Britain and Ireland, 220(2), 217-230.

11. Wallingford, J. B., Niswander, L. A., Shaw, G. M., \& Finnell, R. H. (2013). The continuing challenge of understanding, preventing, and treating neural tube defects. Science, 339(6123).

12. Wilde, J. J., Petersen, J. R., \& Niswander, L. (2014). Genetic, epigenetic, and environmental contributions to neural tube closure. Annual review of genetics, 48, 583-611.

13. Greene, N. D., Stanier, P., \& Copp, A. J. (2009). Genetics of human neural tube defects. Human molecular genetics, 18(R2), R113-R129.

14. Sudiwala, S., Palmer, A., Massa, V., Burns, A. J., Dunlevy, L. P., De Castro, S. C., .. \& Greene, N. D. (2019). Cellular mechanisms underlying Pax3-related neural tube defects and their prevention by folic acid. Disease models \& mechanisms, 12(11), dmm042234.

15. Torban, E., Wang, H. J., Groulx, N., \& Gros, P. (2004). Independent mutations in mouse Vangl2 that cause neural tube defects in looptail mice impair interaction with members of the Dishevelled family. Journal of Biological Chemistry, 279(50), 52703-52713.

16. Gray, J. D., Kholmanskikh, S., Castaldo, B. S., Hansler, A., Chung, H., Klotz, B., .. \& Ross, M. E. (2013). LRP6 exerts non-canonical effects on Wnt signaling during neural tube closure. Human molecular genetics, 22(21), 4267-4281.

17. Carter, M., Ulrich, S., Oofuji, Y., Williams, D. A., \& Elizabeth Ross, M. (1999). Crooked tail (Cd) models human folate-responsive neural tube defects. Human molecular genetics, 8(12), 2199-2204.

18. Wilde, J. J., Petersen, J. R., \& Niswander, L. (2014). Genetic, epigenetic, and environmental contributions to neural tube closure. Annual review of genetics, 48, 583-611.

19. Chen, Z., Lei, Y., Cao, X., Zheng, Y., Wang, F., Bao, Y., .. \& \& Wang, H. (2018). Genetic analysis of Wnt/PCP genes in neural tube defects. BMC Medical Genomics, 11(1), 1-9.

20. Murdoch, J. N., \& Copp, A. J. (2010). The relationship between sonic Hedgehog signaling, cilia, and neural tube defects. Birth Defects Research Part A: Clinical and Molecular Teratology, 88(8), 633-652.

21. Kim, J., Lei, Y., Guo, J., Kim, S. E., Wlodarczyk, B. J., Cabrera, R. M., .. \& Finnell, R. H. (2018). Formate rescues neural tube defects caused by mutations in Slc25a32. Proceedings of the National Academy of Sciences, 115(18), 4690-4695.

22. Copp, A. J., \& Greene, N. D. (2010). Genetics and development of neural tube defects. The Journal of Pathology: A Journal of the Pathological Society of Great Britain and Ireland, 220(2), 217-230. 
23. Bettegowda, C., Agrawal, N., Jiao, Y., Sausen, M., Wood, L. D., Hruban, R. H., .. \& Kinzler, K. W. (2011). Mutations in CIC and FUBP1 contribute to human oligodendroglioma. Science, 333(6048), $1453-1455$.

24. Roch, F., Jiménez, G., \& Casanova, J. (2002). EGFR signalling inhibits Capicua-dependent repression during specification of Drosophila wing veins.

25. Jiménez, G., Shvartsman, S. Y., \& Paroush, Z. E. (2012). The Capicua repressor-a general sensor of RTK signaling in development and disease. Journal of cell science, 125(6), 1383-1391.

26. Astigarraga, S., Grossman, R., Díaz-Delfin, J., Caelles, C., Paroush, Z. E., \& Jimenez, G. (2007). A MAPK docking site is critical for downregulation of Capicua by Torso and EGFR RTK signaling. The EMBO journal, 26(3), 668-677.

27. Wong, D., \& Yip, S. (2020). Making heads or tails-the emergence of capicua (CIC) as an important multifunctional tumour suppressor. The Journal of pathology, 250(5), 532-540.

28. Huang, S. C., Zhang, L., Sung, Y. S., Chen, C. L., Kao, Y. C., Agaram, N. P., .. \& Antonescu, C. R. (2016). Recurrent CIC gene abnormalities in angiosarcomas: a molecular study of 120 cases with concurrent investigation of PLCG1, KDR, MYC, and FLT4 gene alterations. The American journal of surgical pathology, 40(5), 645.

29. Lam, Y. C., Bowman, A. B., Jafar-Nejad, P., Lim, J., Richman, R., Fryer, J. D., .. \& Zoghbi, H. Y. (2006). ATAXIN-1 interacts with the repressor Capicua in its native complex to cause SCA1 neuropathology. Cell, 127(7), 1335-1347.

30. Lu, H. C., Tan, Q., Rousseaux, M. W., Wang, W., Kim, J. Y., Richman, R., .. \& Zoghbi, H. Y. (2017). Disruption of the ATXN1-CIC complex causes a spectrum of neurobehavioral phenotypes in mice and humans. Nature genetics, 49(4), 527-536.

31. Cao, X., Wolf, A., Kim, S. E., Cabrera, R. M., Wlodarczyk, B. J., Zhu, H., .. \& \& Lei, Y. (2021). CIC de novo loss of function variants contribute to cerebral folate deficiency by downregulating FOLR1 expression. Journal of Medical Genetics, 58(7), 484-494.

32. Wolujewicz, P., Aguiar-Pulido, V., AbdelAleem, A., Nair, V., Thareja, G., Suhre, K., .. \& Ross, M. E. (2021). Genome-wide investigation identifies a rare copy-number variant burden associated with human spina bifida. Genetics in Medicine, 1-8.

33. Van der Auwera, G. A., Carneiro, M. O., Hartl, C., Poplin, R., Del Angel, G., Levy-Moonshine, A., .. \& \& DePristo, M. A. (2013). From FastQ data to high-confidence variant calls: the genome analysis toolkit best practices pipeline. Current protocols in bioinformatics, 43(1), 11-10.

34. Li, H., \& Durbin, R. (2009). Fast and accurate short read alignment with Burrows-Wheeler transform. bioinformatics, 25(14), 1754-1760.

35. Poplin, R., Chang, P. C., Alexander, D., Schwartz, S., Colthurst, T., Ku, A., .. \& DePristo, M. A. (2018). A universal SNP and small-indel variant caller using deep neural networks. Nature biotechnology, 36(10), 983-987.

36. McLaren, W., Gil, L., Hunt, S. E., Riat, H. S., Ritchie, G. R., Thormann, A., .. \& Cunningham, F. (2016). The ensembl variant effect predictor. Genome biology, 17(1), 1-14.

37. Jay, J. J., \& Brouwer, C. (2016). Lollipops in the clinic: information dense mutation plots for precision medicine. PloS one, 11(8), e0160519.

38. Rothenberg, S. P., da Costa, M. P., Sequeira, J. M., Cracco, J., Roberts, J. L., Weedon, J., \& Quadros, E. V. (2004). Autoantibodies against folate receptors in women with a pregnancy complicated by a neural-tube defect. New England Journal of Medicine, 350(2), 134-142.

39. Cabrera, R. M., Shaw, G. M., Ballard, J. L., Carmichael, S. L., Yang, W., Lammer, E. J., \& Finnell, R. H. (2008). Autoantibodies to folate receptor during pregnancy and neural tube defect risk. Journal of reproductive immunology, 79(1), 85-92.

40. Ramaekers, V. T., Rothenberg, S. P., Sequeira, J. M., Opladen, T., Blau, N., Quadros, E. V., \& Selhub, J. (2005). Autoantibodies to folate receptors in the cerebral folate deficiency syndrome. New England Journal of Medicine, 352(19), 1985-1991.

41. Copp, A. J., Greene, N. D., \& Murdoch, J. N. (2003). The genetic basis of mammalian neurulation. Nature Reviews Genetics, 4(10), 784-793. 
42. Wallingford, J. B. (2006). Planar cell polarity, ciliogenesis and neural tube defects. Human molecular genetics, 15(suppl_2), R227-R234.

43. Wallingford, J. B. (2012). Planar cell polarity and the developmental control of cell behavior in vertebrate embryos. Annual review of cell and developmental biology, 28, 627-653.

44. Murdoch, J. N., Damrau, C., Paudyal, A., Bogani, D., Wells, S., Greene, N. D., .. \& Copp, A. J. (2014). Genetic interactions between planar cell polarity genes cause diverse neural tube defects in mice. Disease models \& mechanisms, 7(10), 1153-1163.

45. Kibar, Z., Torban, E., McDearmid, J. R., Reynolds, A., Berghout, J., Mathieu, M., .. \& Gros, P. (2007). Mutations in VANGL1 associated with neural-tube defects. New England Journal of Medicine, 356(14), 1432-1437.

46. Lei, Y. P., Zhang, T., Li, H., Wu, B. L., Jin, L., \& Wang, H. Y. (2010). VANGL2 mutations in human cranial neural-tube defects. New England Journal of Medicine, 362(23), 2232-2235.

47. Kibar, Z., Salem, S., Bosoi, C. M., Pauwels, E., De Marco, P., Merello, E., .. \& Gros, P. (2011). Contribution of VANGL2 mutations to isolated neural tube defects. Clinical genetics, 80(1), 76-82.

48. Tian, T., Lei, Y., Chen, Y., Karki, M., Jin, L., Finnell, R. H., . . \& Ren, A. (2020). Somatic mutations in planar cell polarity genes in neural tissue from human fetuses with neural tube defects. Human genetics, 139(10), 1299-1314.

49. Rousseaux, M. W., Tschumperlin, T., Lu, H. C., Lackey, E. P., Bondar, V. V., Wan, Y. W., .. \& Orr, H. T. (2018). ATXN1-CIC complex is the primary driver of cerebellar pathology in spinocerebellar ataxia type 1 through a gain-of-function mechanism. Neuron, 97(6), 1235-1243.

50. Yang, R., Chen, L. H., Hansen, L. J., Carpenter, A. B., Moure, C. J., Liu, H., .. \& Yan, H. (2017). Cic loss promotes gliomagenesis via aberrant neural stem cell proliferation and differentiation. Cancer research, 77(22), 6097-6108.

51. Hwang, I., Pan, H., Yao, J., Elemento, O., Zheng, H., \& Paik, J. (2020). CIC is a critical regulator of neuronal differentiation. JCI insight, 5(9).

52. Piedrahita, J. A., Oetama, B., Bennett, G. D., Van Waes, J., Kamen, B. A., Richardson, J., ... \& Finnell, R. H. (1999). Mice lacking the folic acid-binding protein Folbp1 are defective in early embryonic development. Nature genetics, 23(2), 228-232.

53. Steinfeld, R., Grapp, M., Kraetzner, R., Dreha-Kulaczewski, S., Helms, G., Dechent, P., .. \& \& Gartner, J. (2009). Folate receptor alpha defect causes cerebral folate transport deficiency: a treatable neurodegenerative disorder associated with disturbed myelin metabolism. The American Journal of Human Genetics, 85(3), 354-363.

54. Rothenberg, S. P., da Costa, M. P., Sequeira, J. M., Cracco, J., Roberts, J. L., Weedon, J., \& Quadros, E. V. (2004). Autoantibodies against folate receptors in women with a pregnancy complicated by a neural-tube defect. New England Journal of Medicine, 350(2), 134-142.

55. Saitsu, H. (2017). Folate receptors and neural tube closure. Congenital anomalies, 57(5), 130-133.

56. Findley, T. O., Tenpenny, J. C., O’Byrne, M. R., Morrison, A. C., Hixson, J. E., Northrup, H., \& Au, K. S. (2017). Mutations in folate transporter genes and risk for human myelomeningocele. American Journal of Medical Genetics Part A, 173(11), 2973-2984.

57. Tada, M., \& Heisenberg, C. P. (2012). Convergent extension: using collective cell migration and cell intercalation to shape embryos. Development, 139(21), 3897-3904.

58. Butler, M. T., \& Wallingford, J. B. (2018). Spatial and temporal analysis of PCP protein dynamics during neural tube closure. Elife, 7, e36456.

59. Blankenship, J. T., Backovic, S. T., Sanny, J. S., Weitz, O., \& Zallen, J. A. (2006). Multicellular rosette formation links planar cell polarity to tissue morphogenesis. Developmental cell, 11(4), 459-470.

60. Butler, M. T., \& Wallingford, J. B. (2017). Planar cell polarity in development and disease. Nature reviews Molecular cell biology, 18(6), 375-388.

61. Humphries, A. C., Narang, S., \& Mlodzik, M. (2020). Mutations associated with human neural tube defects display disrupted planar cell polarity in Drosophila. Elife, 9, e53532.

62. Wang, L., Xiao, Y., Tian, T., Jin, L., Lei, Y., Finnell, R. H., \& Ren, A. (2018). Digenic variants of planar cell polarity genes in human neural tube defect patients. Molecular genetics and metabolism, 124(1), 
94-100.

63. Kibar, Z., Vogan, K. J., Groulx, N., Justice, M. J., Underhill, D. A., \& Gros, P. (2001). Ltap, a mammalian homolog of Drosophila Strabismus/Van Gogh, is altered in the mouse neural tube mutant Loop-tail. Nature genetics, 28(3), 251-255.

64. Torban, E., Patenaude, A. M., Leclerc, S., Rakowiecki, S., Gauthier, S., Andelfinger, G., ... \& Gros, P. (2008). Genetic interaction between members of the Vangl family causes neural tube defects in mice. Proceedings of the National Academy of Sciences, 105(9), 3449-3454.

65. Wang, J., Hamblet, N. S., Mark, S., Dickinson, M. E., Brinkman, B. C., Segil, N., .. \& WynshawBoris, A. (2006). Dishevelled genes mediate a conserved mammalian PCP pathway to regulate convergent extension during neurulation. Development, 133(9), 1767-1778.

66. Ybot-Gonzalez, P., Savery, D., Gerrelli, D., Signore, M., Mitchell, C. E., Faux, C. H., ... \& Copp, A. J. (2007). Convergent extension, planar-cell-polarity signalling and initiation of mouse neural tube closure. Development, 134(4): 789-799.

67. Curtin, J. A., Quint, E., Tsipouri, V., Arkell, R. M., Cattanach, B., Copp, A. J., .. \& Murdoch, J. N. (2003). Mutation of Celsr1 disrupts planar polarity of inner ear hair cells and causes severe neural tube defects in the mouse. Current Biology, 13(13), 1129-1133.

68. Mohd-Zin, S. W., Marwan, A. I., Abou Chaar, M. K., Ahmad-Annuar, A., \& Abdul-Aziz, N. M. (2017). Spina bifida: pathogenesis, mechanisms, and genes in mice and humans. Scientifica, 2017.

69. Juriloff, D. M., \& Harris, M. J. (2012). A consideration of the evidence that genetic defects in planar cell polarity contribute to the etiology of human neural tube defects. Birth Defects Research Part A: Clinical and Molecular Teratology, 94(10), 824-840.

70. Oishi, A., Makita, N., Sato, J., \& Iiri, T. (2012). Regulation of RhoA signaling by the cAMP-dependent phosphorylation of RhoGDI $\alpha$. Journal of Biological Chemistry, 287(46), 38705-38715.

71. Balashova, O. A., Visina, O., \& Borodinsky, L. N. (2017). Folate receptor 1 is necessary for neural plate cell apical constriction during Xenopus neural tube formation. Development, 144(8), 1518-1530.

Table1 Information of the detected CIC rare missense variants in infants with NTDs

Figure 1 Identification of CIC rare missense variants in infants with NTDs. (A) Protein amino acid locus of CIC missense variants. (B) Amino Acid conservation of identified variants among different species.

Figure 2 Subcellular localization and protein abundance of CIC wildtype and CIC variants. (A) Hela cells were transfected with mutated and wildtype constructs of GFP-tagged CIC and pEGFP backbone vector for $36 \mathrm{~h}$ and were imaged under deconvolution microscope. Scale bar 5um. (B) Western Blotting was performed in Hela cells $48 \mathrm{~h}$ after transfection. GAPDH was used as loading control. (C) Western Blotting was repeated for three times and student t-test was performed to compare the protein level between wildtype and mutant.

Figure 3 Overexpression of CIC mutants affected FOLR1 protein level and folate binding ability of Hela cells. (A) Hela cells were transfected with mutated and wildtype constructs of GFP-tagged CIC and pEGFP backbone vector for $48 \mathrm{~h}$, and western blotting was performed to quantify FOLR1 protein level in each group. GAPDH was used as loading control. (B) Western blotting was repeated for three times and Student's ttest was performed to compare the protein level between wildtype and mutant. (C) Folate of Hela cells transfected with CIC wildtype and variants were collected and quantified in triplicates.

Figure 4 Overexpression of CIC mutants affected core PCP protein Vangl2 and its downstream protein RhoA in Hela cells. (A) Hela cells were transfected with mutated and wildtype constructs of GFP-tagged CIC and pEGFP backbone vector for 48h, and western blotting was performed to quantify Vangl2 and RhoA protein level in each group. GAPDH was used as loading control. (B) Western blotting was repeated for three times and Student's t-test was performed to compare Vangl2 and RhoA protein level between wildtype and mutant.

Figure 5 CIC loss of function diminished core PCP protein Vangl2 and its downstream protein RhoA in NIH3T3 cells. (A) NIH3T3 cells were transfected with CIC wildtype, CIC-R353X construct and pEGFP backbone vector for $48 \mathrm{~h}$, and Western Blotting was performed to quantify GFP-CIC, Vangl2 and RhoA 
protein level in each group. GAPDH was used as loading control. (B) Western Blotting was repeated for three times and student t-test was performed to compare Vangl2 and RhoA protein level between wildtype and R353X.

\section{Hosted file}

Table.docx available at https://authorea.com/users/448630/articles/547411-cic-missensevariants-contribute-to-susceptibility-for-spina-bifida

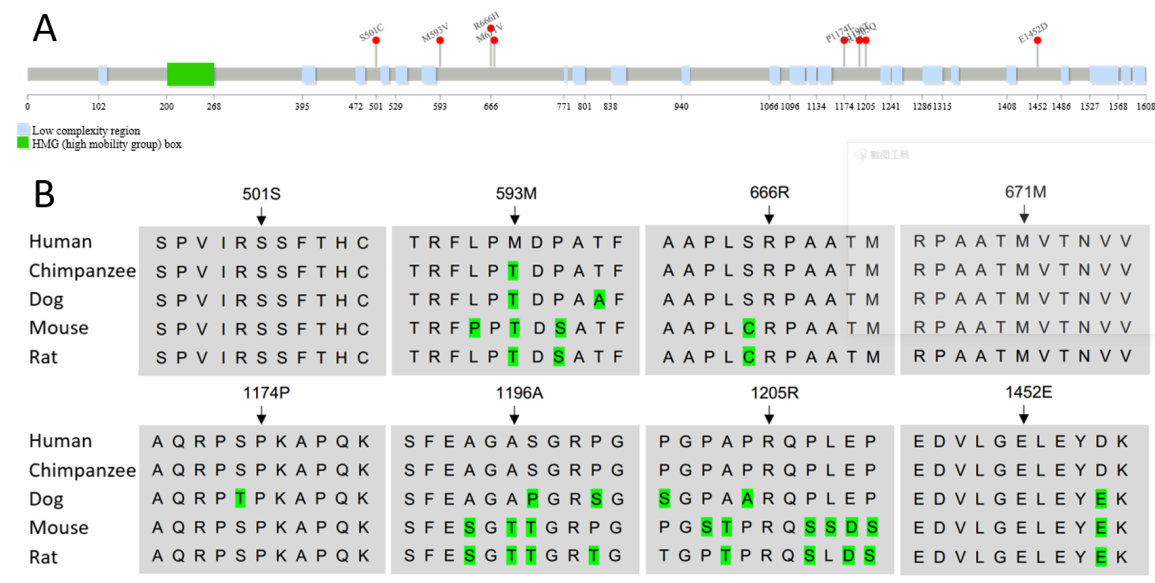

A WT S501C M593V R666H M671V P1174L A1196T R1205Q E1452D PEGFP

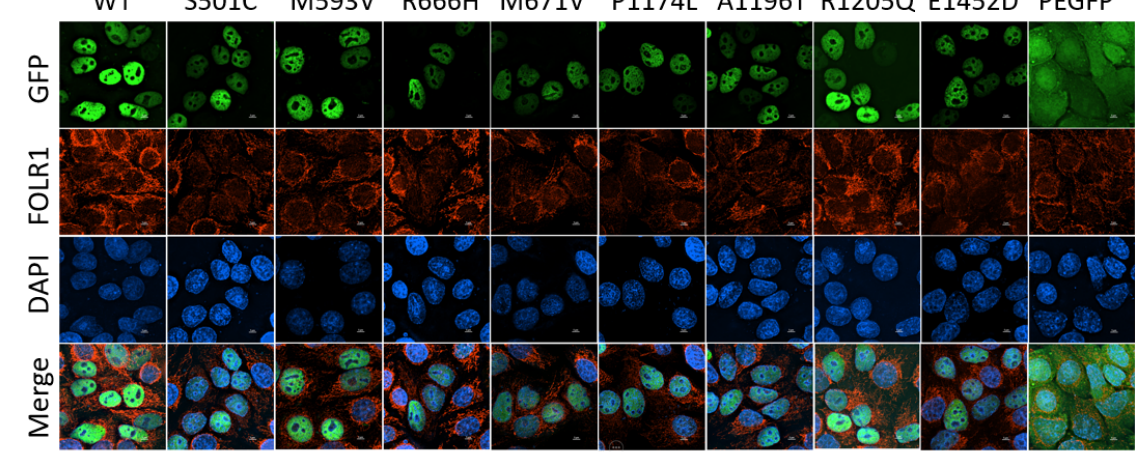

B

C
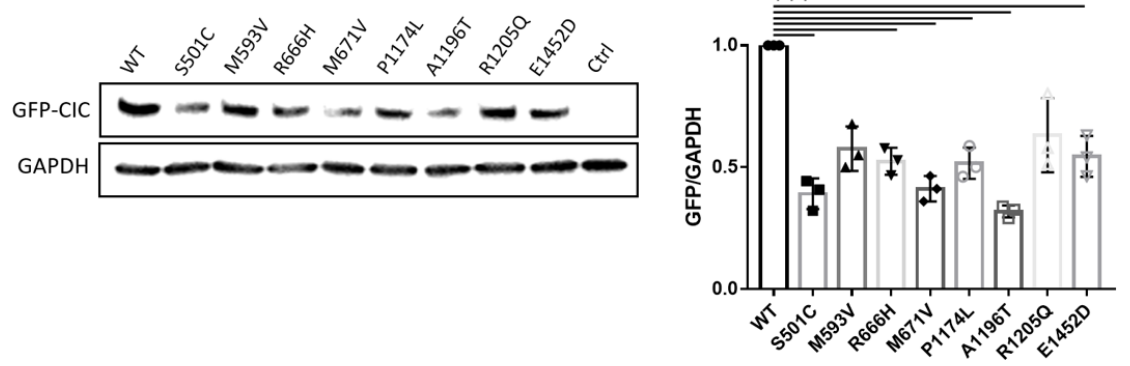


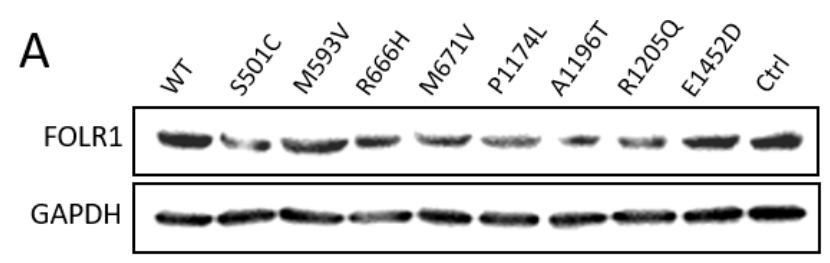

B

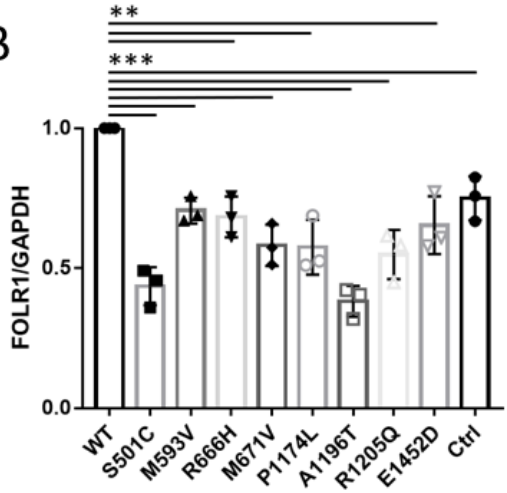

C

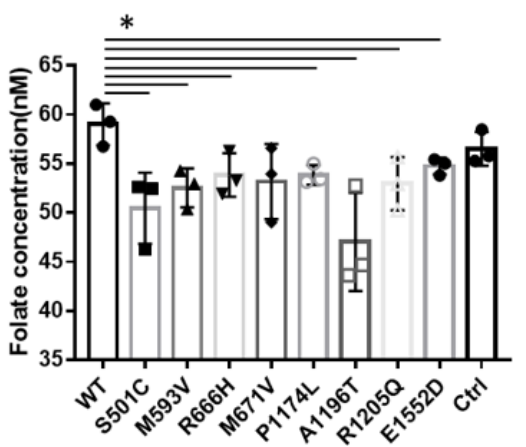

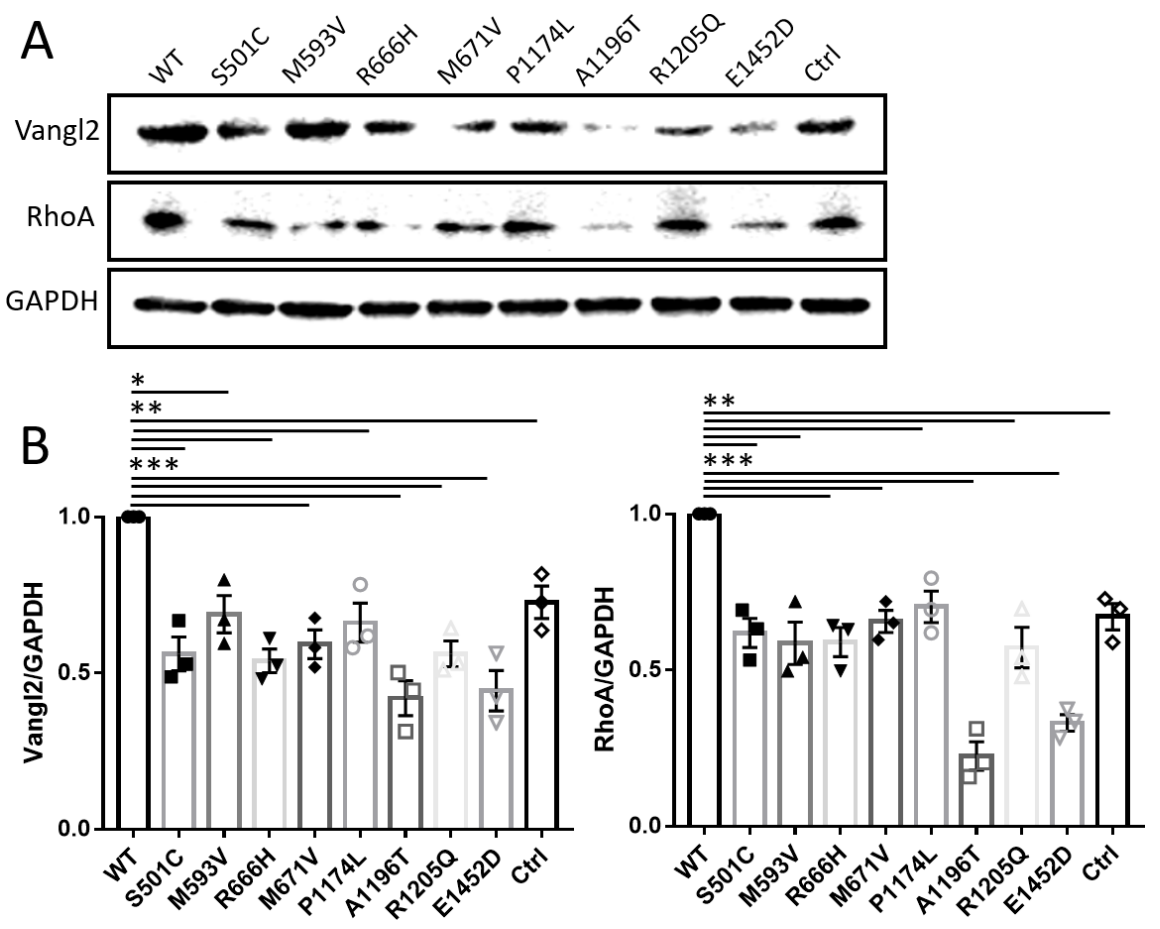




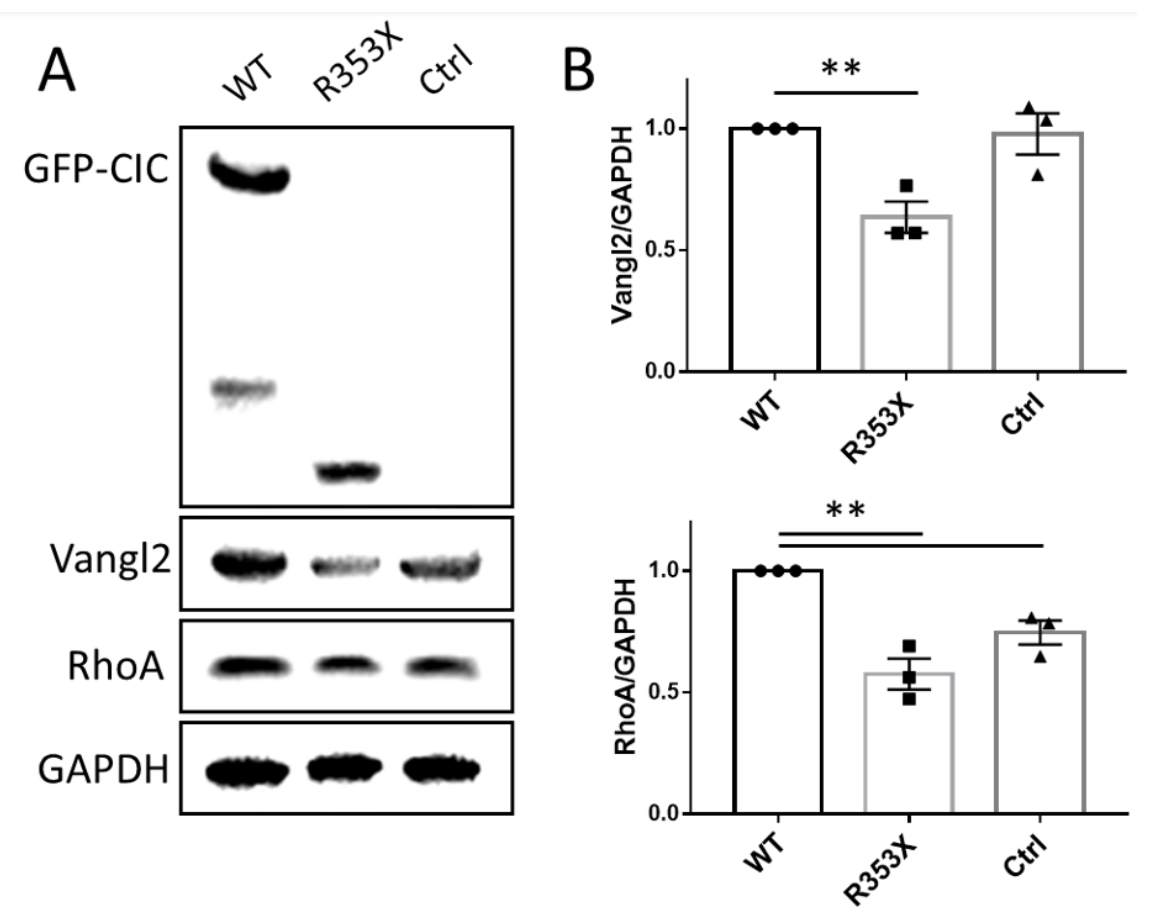

